\title{
Hydrogen Portal to Exotic Radioactivity
}

\author{
David McKeen, ${ }^{1, *}$ Maxim Pospelov, ${ }^{2,3, \dagger}$ and Nirmal Raj ${ }^{1, \$}$ \\ ${ }^{1}$ TRIUMF, 4004 Wesbrook Mall, Vancouver, British Columbia V6T 2A3, Canada \\ ${ }^{2}$ School of Physics and Astronomy, University of Minnesota, Minneapolis, Minnesota 55455, USA \\ ${ }^{3}$ William I. Fine Theoretical Physics Institute, School of Physics and Astronomy, University of Minnesota, \\ Minneapolis, Minnesota 55455, USA
}

(Received 6 July 2020; accepted 21 October 2020; published 1 December 2020)

\begin{abstract}
We show that in a special class of dark sector models, the hydrogen atom can serve as a portal to new physics, through its decay occurring in abundant populations in the Sun and on Earth. The large fluxes of hydrogen decay daughter states can be detected via their decay or scattering. By constructing two models for either detection channel, we show that the recently reported excess in electron recoils at XENON1T could be explained by such signals in large regions of parameter space unconstrained by proton and hydrogen decay limits.
\end{abstract}

DOI: 10.1103/PhysRevLett.125.231803

Introduction.-The extremely successful experimental program of building ever larger and cleaner dark matter search experiments based on two-phase xenon has led to extremely important advances in particle astrophysics. Many dark matter models, including both heavy and light particles, are considerably constrained or excluded. At the same time, these experiments-by their sheer size and low levels of contamination-prove to be universal tools in detecting any 1-100 keV energy deposition. Reaching counting rates as low as $O(10-100) / \mathrm{ton} / \mathrm{yr} / \mathrm{keV}$, the XENON1T experiment has shown itself to be a leader in probing rare processes in the energy domain inaccessible to conventional neutrino experiments.

In addition to setting important constraints, the XENON1T experiment has observed an excess over known backgrounds in electron recoils in $2-3 \mathrm{keV}$ energies in 0.65 ton-year of data [1]. Putative explanations proposed include an unmeasured tritium background [1,2] (or any other missed radioactive background in the $\mathrm{keV}$ range), and physics scenarios beyond the standard model (SM) $[1,3,4]$. These papers explore a diverse set of ideas on how the unforeseen $\mathrm{keV}$-scale events may emerge in various new physics scenarios. The following broad picture has emerged with respect to the explanation of the excess thus far: (i) At the moment, there is no clear model of light sub$\mathrm{keV}$ particles that would be emitted from the Sun, and absorbed by the detector without running into other problems such as astrophysical energy loss constraints.

Published by the American Physical Society under the terms of the Creative Commons Attribution 4.0 International license. Further distribution of this work must maintain attribution to the author(s) and the published article's title, journal citation, and DOI. Funded by SCOAP . (ii) Light neutrinos, produced in the Sun, Earth or reactors, cannot be the source of the signal without deviating from the conventional SM physics. Even then many scenarios involving novel interactions such as, e.g., light neutrino magnetic moments are again ruled out by stellar energy loss constraints and/or cosmology. (iii) So far the dark matter route seems to be theoretically the easiest avenue for arranging excess events. Ideas include absorption of $\mathrm{keV}$ scale dark matter, inelastic dark matter deexciting in the detector, or elastic scattering of a dark matter subcomponent that moves faster than expected virial Galactic velocities. In many cases the astrophysics constraints can be relaxed or avoided because the dark matter particles are heavy enough, while the absorption of light $\mathrm{keV}$ dark matter can also be safe in many scenarios due to extremely large number densities, and correspondingly tiny couplings of such particles.

In this Letter we explore the idea of radioactivity induced by standard hydrogen in models where the electron and proton couple to exotic particles. This may be viewed as an alternative to using dark matter as a source of keV energy. Because of the $\mathrm{GeV}$-scale mass of daughter particles many of the astrophysics constraints are irrelevant, while hydrogen is guaranteed to be an abundant source, both on Earth and in the Sun, even after taking into account strong constraints on its lifetime.

It is known that dark matter decays may lead to a substantial flux of particles at Earth's location, with the main sources of the flux being the local Milky Way halo as well as the global dark matter density in the Universe [5]. For a maximum allowed abundance of dark matter particles, and shortest lifetime scales (exceeding the lifetime of the Universe $\tau_{U}$ by a factor of $\sim 10$ ), the flux of daughter particles can achieve substantial values and even be comparable with the solar or Earth neutrino fluxes, or 
even those of the cosmic neutrino background [6]. Moreover, even the weak-strength interactions of daughter products could be detected using the most sensitive neutrino and dark matter detectors, existent or in development $[5,6]$. While the decaying dark matter scenario is full of "unknown unknowns," their number shrinks if we get to possible decays of SM particles. The most abundant longlived particle with appreciable mass that may decay without violating charge conservation is the proton. The maximum flux of daughter particles for $O(1)$ multiplicity can be estimated as $N_{\mathrm{p}}^{\odot} \tau_{p}^{-1}\left(4 \pi \mathrm{A}_{\mathrm{U}} \mathrm{U}^{2}\right)^{-1}$, where $\tau_{p}$ is the experimental limit on the proton lifetime and $N_{p}^{\odot}$ is the total number of protons in the Sun. An interesting caveat that has been discussed in recent literature is that if the masses of new physics daughter particles lie close to $m_{p, n}$, the proton itself may be stable [7] but the hydrogen atom could decay [8]. Although relatively strong bounds can be derived on the lifetime of $\mathrm{H}$ using the sensitivity to the final state photon in the radiative capture of $e^{-}$by $p$ [9], these bounds are in fact many orders of magnitude milder than $\tau_{p}$ limits. To summarize, one has the following picture of the maximum attainable fluxes of daughter particles (assuming $O(1)$ multiplicities):

$$
\begin{aligned}
\Phi_{\mathrm{DM} \text { decay }}^{\text {global+MW }} & \sim 10^{4} \mathrm{~cm}^{-2} \mathrm{~s}^{-1}\left(\frac{10 \tau_{U}}{\tau_{\mathrm{DM}}}\right)\left(\frac{1 \mathrm{GeV}}{m_{\mathrm{DM}}}\right), \\
\Phi_{\text {proton decay }}^{\odot} & \sim 10^{-8} \mathrm{~cm}^{-2} \mathrm{~s}^{-1}\left(\frac{10^{30} \mathrm{yr}}{\tau_{p}}\right), \\
\Phi_{\mathrm{H} \text { decay }}^{\odot} & \sim 10^{3} \mathrm{~cm}^{-2} \mathrm{~s}^{-1}\left(\frac{10^{28} \mathrm{~s}}{\tau_{\mathrm{H}}}\right), \\
\Phi_{\mathrm{H} \text { decay }}^{\oplus} & \sim 1 \mathrm{~cm}^{-2} \mathrm{~s}^{-1}\left(\frac{10^{28} \mathrm{~s}}{\tau_{\mathrm{H}}}\right) .
\end{aligned}
$$

All of these decays, and we will concentrate on that of $\mathrm{H}$, can be considered as portals to a new sector that may have additional interactions with the SM, and reveal themselves via scattering or decay. Motivated by recent XENON1T results, we will focus on sub-10 keV energy deposition, while in principle $\mathrm{H}$-portal couplings could lead to up to few $100 \mathrm{MeV}$ energy release. We investigate the detection of large fluxes of daughter states of $\mathrm{H}$ decay occurring in both the Earth and Sun. In particular we explore the two scenarios summarized in Fig. 1: the daughter states could either decay to a photon final state or scatter on electrons in the detector. We show that in both scenarios there exist large regions of parameter space that result in the right flux and energy deposition in XENON1T to fit the recent excess well. From a more conservative standpoint, independent of this particular excess, sensitive neutrino and direct dark matter detection experiments can be used to probe properties of particles emerging from $\mathrm{H}$ decays.

Terestrial $\mathrm{H}$ decay to metastable state.-We first consider a model with the neutron portal, in which the neutron

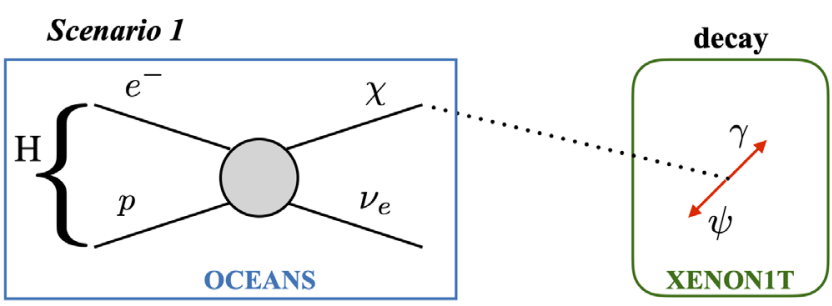

Scenario 2

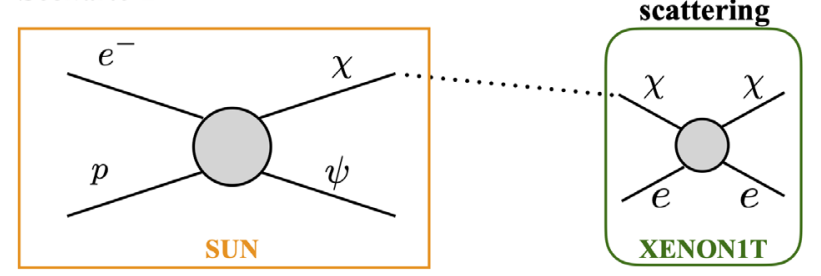

FIG. 1. The two scenarios considered in this work that could explain the XENON1T electron recoil excess. In the first, $\mathrm{H}$ atoms decay on Earth (primarily in the oceans) to produce a dark baryon that propagates through the Earth and decays in a detector to another fermion and a photon. In the second, $e^{-} p$ capture in the Sun produces a fast-moving long-lived dark sector particle that scatters on detector electrons.

mixes with a dark neutron $\chi$ (that may be elementary or composite) $[7,10,11]$,

$$
\mathcal{L} \supset \delta(\bar{n} \chi+\bar{\chi} n)
$$

with mixing angle $\theta=\delta /\left(m_{n}-m_{\chi}\right)$. This model has received much theoretical and experimental attention in connection with the neutron lifetime discrepancy $[8,12]$ as well as potential connections to dark matter $[7,13]$. Note that explanations of the neutron lifetime anomaly that rely on an exotic neutron decay mode could introduce tension with the predicted neutron $\beta$-decay rate using up-to-date measurements of the nucleon axial vector coupling [14] while this could be avoided with oscillations into a mirror sector [15]. If $m_{\chi}<m_{\mathrm{H}} \simeq m_{p}+m_{e}$, atomic hydrogen decays to $\chi+\nu_{e}$ with a lifetime $[8,9]$

$$
\tau_{\mathrm{H}}=10^{29} \mathrm{~s}\left(\frac{10^{-10}}{\theta}\right)^{2}\left(\frac{m_{e}}{Q}\right)^{2},
$$

where $Q=m_{\mathrm{H}}-m_{\gamma}$. In this model the hydrogen lifetime through this channel is constrained to be $\tau_{\mathrm{H}} \gtrsim 10^{29} \mathrm{~s}$ by a search for $n \rightarrow \chi \gamma$ [16] and a recast of Borexino data [17] to constrain $H \rightarrow \chi \nu \gamma$ [9], displayed in the top panel of Fig. 2. There is also a lower limit on the $\chi$ mass of $m_{\chi}>938.0 \mathrm{MeV}$ from the stability of ${ }^{9} \mathrm{Be}[7,18]$. Given this bound, the speed of $\chi$ 's produced in $\mathrm{H}$ decay is $v_{\chi} / c \simeq Q / m_{\chi}<0.8 \times 10^{-3}$. This is lower than the Sun's surface escape velocity of $0.002 c$, which can be overcome only by the energetic tail of the Boltzmann distribution. Therefore for detecting $\chi$ on Earth, we must consider terrestrial sources of hydrogen. One simple possibility to 


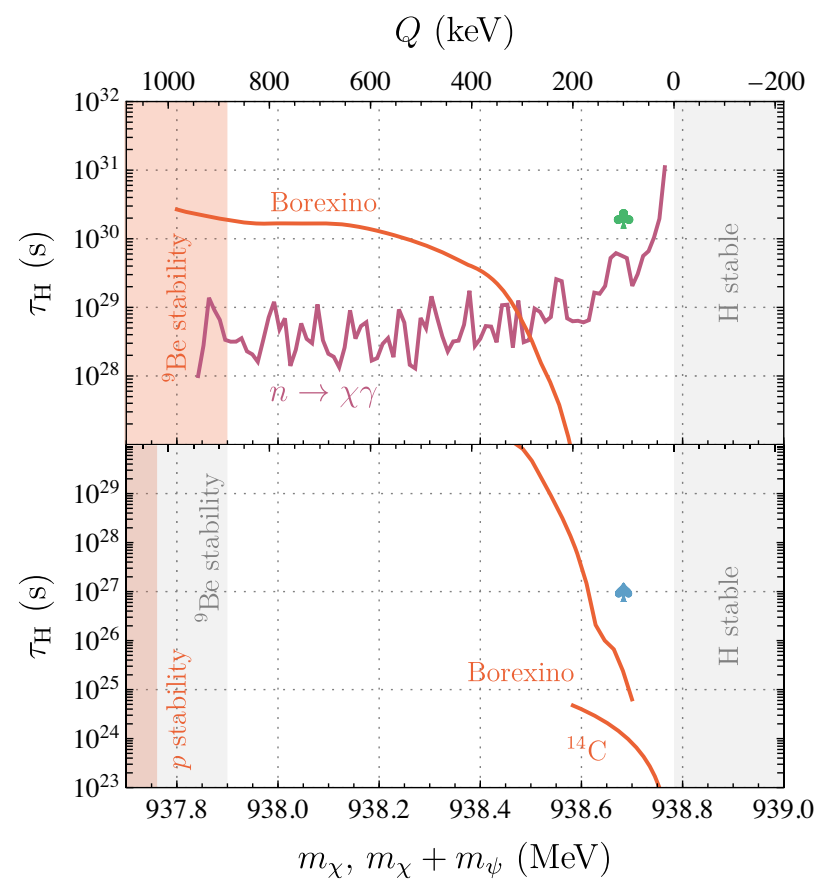

FIG. 2. Lower limits, as derived in Ref. [9], on the H lifetime as functions of $Q$ and the total mass of the decay final state in our two scenarios. Top: Limits from the low energy Borexino spectrum [17] (red) and the search for $n \rightarrow \chi \gamma$ [16] (purple) in the neutron-mixing model of scenario 1. Bottom: Limits from Ref. [17] and a study of ${ }^{14} \mathrm{C}$ purity [20] on the EFT interaction of scenario 2. Also shown with a green club and blue spade are the benchmark $\left(\tau_{\mathrm{H}}, Q\right)$ in scenario 1 and 2 , respectively, that give rise to the spectra in Fig. 3 .

detect $\chi$ 's after production involves a slight extension of the model: we add a neutral fermion $\psi$, and a transition magnetic dipole moment between $\chi$ and $\psi$ (Indeed we expect that the dark neutron $\chi$ has additional interactions because of constraints from neutron stars [19] as well as inheriting a magnetic moment from the neutron. We also assume here that $n-\psi$ mixing is smaller than that of $n-\chi$.),

$$
\mathcal{L} \supset \frac{1}{\Lambda_{d}} \bar{\psi} \sigma^{\mu \nu} F_{\mu \nu} \chi
$$

Here $\Lambda_{d}$ is a new scale which could come from, e.g., nontrivial dynamics in the dark sector such as the exchange of charged particles at the $100 \mathrm{GeV}-\mathrm{TeV}$ scale. For our purposes, we remain agnostic about the details of its origin. If $m_{\chi}-m_{\psi} \equiv \delta m>0, \chi$ decays to $\psi$ and a photon of energy $\omega=\delta m$ with the decay length

$$
\begin{aligned}
v_{\chi} \tau_{\chi}= & 6 \times 10^{8} \mathrm{~cm}\left(\frac{3 \mathrm{keV}}{\delta m}\right)^{3} \\
& \times\left(\frac{\Lambda_{d}}{10^{5} \mathrm{GeV}}\right)^{2}\left(\frac{Q}{100 \mathrm{keV}}\right),
\end{aligned}
$$

when produced in $\mathrm{H}$ decay. For $v_{\chi} \tau_{\chi}$ not too small compared to the radius of Earth, $R_{\oplus}=6.4 \times 10^{8} \mathrm{~cm}$, the decay of terrestrial atomic hydrogen can produce photons through $\chi$ decay that can potentially be observable.

The vast majority of atomic hydrogen on Earth is in the "hydrosphere" - the Earth's collection of water-with a total volume of $1.4 \times 10^{9} \mathrm{~km}^{3}$ [21], corresponding to $N_{\mathrm{H}} \simeq 10^{47}$ hydrogen atoms. Modeling the hydrosphere as uniformly covering the Earth's surface, the rate of $\chi$ decays in a detector of volume $\ell_{\text {det }}^{3}$ near the surface is

$$
R=\frac{f_{\mathrm{mol}} N_{\mathrm{H}}}{\tau_{\mathrm{H}}} \frac{\ell_{\mathrm{det}}^{3}}{4 \pi R_{\oplus}^{3}} F\left(\frac{R_{\oplus}}{\ell_{\chi}}, \frac{r_{\mathrm{min}}}{R_{\oplus}}\right) .
$$

In this expression $\ell_{\chi}=\left(1-v_{\chi}^{2}\right)^{-1 / 2} v_{\chi} \tau_{\chi} \simeq v_{\chi} \tau_{\chi}$ is the mean distance traveled by $\chi$ 's produced in $\mathrm{H}$ decay, $r_{\text {min }}$ is the distance from the detector to the nearest point in the hydrosphere, and $f_{\text {mol }}$ characterizes the difference between the atomic and molecular $\mathrm{H}$ lifetimes arising from the distortion of the molecular electron wave function. In molecules with covalent bonds, $f_{\text {mol }}$ is typically an $\mathcal{O}(1)$ number $[9,22,23]$. Lastly, we have defined

$$
F(y, \delta)=\frac{y}{2} \int_{-1}^{1-\delta^{2} / 2} d x \frac{\exp [-y \sqrt{2(1-x)}]}{2(1-x)} .
$$

For $\ell_{\chi} \sim R_{\oplus}, r_{\min }, F$ is $\mathcal{O}(1)$ and an appreciable number of $\chi$ 's produced terrestrially can decay in an experiment on Earth. In a liquid Xe detector such as XENON1T, this gives a photon deposition rate of

$$
R \simeq \frac{140}{\text { ton } \mathrm{yr}}\left(\frac{10^{30} \mathrm{~s}}{\tau_{\mathrm{H}}}\right)\left(\frac{f_{\mathrm{mol}}}{0.5}\right) \times F .
$$

Photons with $\omega \sim \mathrm{keV}$ produced in XENON1T have a short travel time before they initiate photoabsorption that eventually leads to multiple ionization electrons [24]. This estimate shows that in the neutron-mixing model with a value of $\tau_{\mathrm{H}}$ allowed by data and $v_{\chi} \tau_{\chi} \sim R_{\oplus}$ (such that $F$ is not small), hydrogen decay on Earth remarkably produces the right amount of photons at the right energy to explain the XENON1T excess in Ref. [1]. In Fig. 3 we show the total event rate for $\omega=\delta m=2.75 \mathrm{keV}$ photons, with $\tau_{\mathrm{H}}=2 \times 10^{30} \mathrm{~s}, Q=100 \mathrm{keV}, \ell_{\chi}=3 \times 10^{12} \mathrm{~cm}$ (corresponding to $\Lambda_{d}=1.1 \times 10^{5} \mathrm{GeV}$ ), $f_{\text {mol }}=0.5$, and $r_{\min } / R_{\oplus}=0.2$ which produces about 50 events after convolving with the detection efficiencies given in Ref. [1]. Here we account for the detector energy resolution of $0.45 \mathrm{eV}$ [25] by Gaussian-smearing the signal rate. The photon deposition rate for our benchmark point is seen to fit the excess well, borne out by a two-parameter fit giving $\chi^{2} /$ d.o.f. $=0.75$ with a $\Delta \chi^{2}$ of 10.77 with respect to the background model, taking into account the first 14 bins.

Production in the Sun followed by scattering.-Now we consider a signal produced by scattering on electrons, 


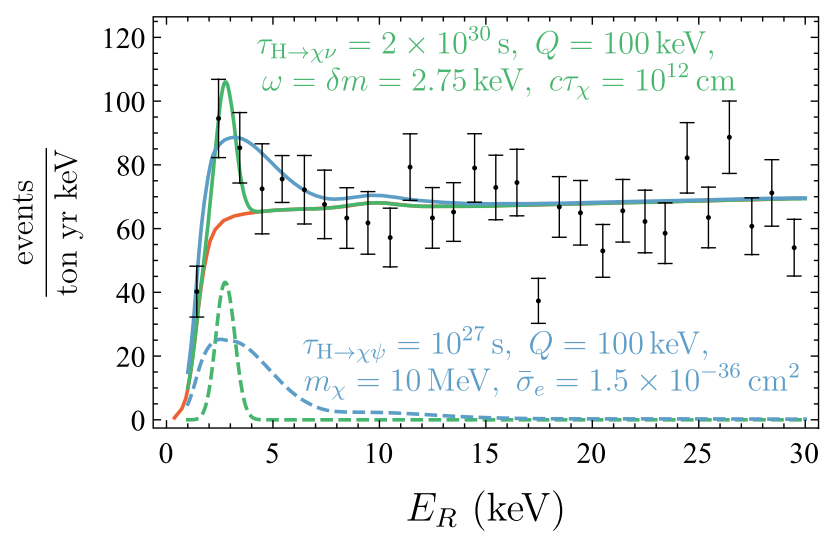

FIG. 3. Event rates in XENON1T in the scenarios of decay (green) and scattering (blue) of hydrogen daughters, seen to significantly reduce tensions in the data for the benchmark points indicated in Fig. 2. Here the red curve denotes the known backgrounds taken from Ref. [1]. The dashed curves depict the signal only while the solid curves depict the signal plus background. See text for further details.

which can recoil with $2-3 \mathrm{keV}$ for light projectiles striking them with velocities $v / c>0.02$. The model above is unsuitable for this signal as $v_{\chi} \lesssim 10^{-3} c$. We thus consider an alternative possibility with light neutral fermions interacting with $e$ and $p$ via a dimension-6 operator:

$$
\mathcal{L}=\frac{1}{\Lambda^{2}}(\bar{\psi} p)(\bar{\chi} e)
$$

Constraints on this interaction come from $p$ decay (and potentially ${ }^{9} \mathrm{Be}$ decay depending on the UV completion), as well as radiative $\mathrm{H}$ decay in Borexino [17,20], all of which are displayed in the bottom panel of Fig. 2. For $m_{\chi}+m_{\psi}<m_{\mathrm{H}}$, this leads to the decay of hydrogen with

$$
\tau_{\mathrm{H}} \simeq 3 \times 10^{27} \mathrm{~s} \sqrt{\frac{m_{e}}{Q}}\left(\frac{\Lambda}{100 \mathrm{PeV}}\right)^{4},
$$

where here, $Q=m_{\mathrm{H}}-m_{\chi}-m_{\psi} \simeq m_{p}+m_{e}-m_{\chi}-m_{\psi}$. A large flux of $\chi$ and $\psi$ states comes from the decay of hydrogen in the Sun (or, more accurately, $e^{-} p$ capture in the stellar plasma), exceeding that of the flux from oceanic $\mathrm{H}$ decay. We assume that $\chi$ scatters on electrons, e.g., through a dimension- 6 operator such as $\bar{\chi} \chi \bar{e} e$, and that the scattered electron gives a measurable signal. (This signal is similar to the scattering of boosted dark matter in noble liquid detectors [26].) Postproduction the $\chi$ and $\psi$ states move at speeds

$v_{\chi}=\sqrt{\frac{2 m_{\psi} Q}{m_{\chi}\left(m_{\chi}+m_{\psi}\right)}}, \quad v_{\psi}=\sqrt{\frac{2 m_{\chi} Q}{m_{\psi}\left(m_{\chi}+m_{\psi}\right)}}$,

assuming $m_{\chi}, m_{\psi} \gg Q$. Taking, for example, $m_{\chi}=10 \mathrm{MeV}$ and $Q=100 \mathrm{keV}$, we get $v_{\chi} / c=0.14$, which, interestingly, provides a good fit to the excess at XENON1T as seen in Ref. [3]. The scattering rate is proportional to the incident flux, which is $O\left(10^{3}\right)$ higher for $e^{-} p$ capture in the Sun than in hydrogen decay in the oceans. The solar flux of $\chi$ is given by [8]

$$
\Phi_{\chi}^{\odot}=\frac{2.5 \times 10^{31}}{\mathrm{~cm}^{2} \tau_{\mathrm{H}}} \sqrt{\frac{10^{7} \mathrm{~K}}{T}}\left(\frac{n_{e}}{2.5 \times 10^{25} \mathrm{~cm}^{-3}}\right),
$$

where the electron number density $n_{e}$ and temperature $T$ have been normalized to their average values in the solar core, taken to have $0.2 R_{\odot}$ radius. The scattering rate of $\chi$ per ton of detector mass is

$$
\frac{d R}{d E_{R}}=N_{\text {ton }} \Phi_{\chi}^{\odot} \frac{d \sigma}{d E_{R}},
$$

where $N_{\text {ton }}$ is the number of target atoms per ton of detector and the differential cross section is given by

$$
\frac{d \sigma}{d E_{R}}=\frac{\bar{\sigma}_{e}}{2 \alpha^{2} m_{e}^{3} v_{\chi}^{2}} \int_{q_{-}}^{q_{+}} d q q K\left(E_{R}, q\right),
$$

where $\bar{\sigma}_{e}$ is the cross section for scattering on unbound electrons at $q=\alpha m_{e}, K$ describes the probability to ionize the atom, and the integration limits are

$$
q_{ \pm}=m_{\chi} v_{\chi} \pm \sqrt{m_{\chi}^{2} v_{\chi}^{2}-2 m_{\chi} E_{R}}
$$

Specifying now to the case of scattering in XENON1T, we take the atomic ionization factor $K$, which for Xe is dominated by the $n=3$ shell, from Refs. [27,28], and assume a unit form factor, corresponding to a mediator with mass exceeding the momentum transfer $q=\mathcal{O}(10-100) \mathrm{keV}$. In Fig. 3 we display the event rate for a $\chi$ flux corresponding to $\tau_{\mathrm{H}}=10^{27} \mathrm{~s}$ and $\bar{\sigma}_{e}=1.5 \times 10^{-36} \mathrm{~cm}^{2}$, seen to ameliorate the anomalous excess. As before, we smear the signal rate with a $0.45 \mathrm{GeV}$ Gaussian energy resolution and account for the detection efficiencies given in Ref. [1]. Using the first 14 bins we get $\chi^{2} /$ d.o.f. $=0.58$ with a two-parameter fit, and $\Delta \chi^{2}=12.81$ with respect to the background model.

Discussion.-Returning to our starting point with the maximum fluxes (1), we note that by virtue of very strong constraints on the proton lifetime, it is unlikely that proton decay could result in a sizable rate for interactions or decays of daughter products in almost any realistic-sized detector. In contrast, it is is clear that dark matter decay could source relatively large interaction rates of its daughter products. In this Letter, we show that hydrogen decay offers a unique window into new sources of exotic radioactivity that have largely gone unstudied. Large numbers of $\mathrm{H}$ atoms exist nearby in the Sun and on Earth, and could lead to appreciable signals in large volume detectors sensitive to energy depositions of $1-100 \mathrm{keV}$. The class of models 
where this could happen requires matching of the total mass of daughter particles to $\mathrm{H}$ mass to within $O(1 \mathrm{MeV})$.

In this Letter we have illustrated this point by explaining the recently reported XENON1T electron recoil excess by $e^{-} p$ capture processes in the oceans and the Sun. In the first scenario, dark neutrons produced in hydrogen atom decays in the oceans in turn decay in-flight to a photon final state that deposits 2-3 keV energy in XENON1T. In the second scenario, long-lived dark sector states produced in $e^{-} p$ capture in the Sun travel at speeds $\gg 10^{-3} c$ and scatter on electrons in XENON1T. This scenario could also accommodate a signal from the decay of the dark sector particles produced in $e^{-} p$ capture, but we have not pursued this possibility. The event rates in our model, displayed in Fig. 3, provide a prediction for the event counts to expect at the future XENONnT experiment [29] with $\mathcal{O}(10)$ higher exposure than XENON1T, as well as at PANDAX-II [30], LZ [31], and DARKSIDE [32].

We note that the solar flux in Eq. (12) scales as $T^{-1 / 2}$ due to Sommerfeld enhancement effects, whereas the flux of light boson states that contribute to stellar cooling are generally increasing functions of the temperature. In this setup, $\mathrm{H}$ decay in the sun contributes about one part in $10^{7}$ of the solar power radiated. Moreover, constraints on light bosons from the cooling of red giants and horizontal branch stars are altogether avoided in this setup since the helium cores of these stars cannot decay due to nuclear binding energy. Further study of these and related models, particularly their effects on cosmology, would certainly be interesting.

D. M. and N.R. are supported in part by the Natural Sciences and Engineering Research Council of Canada (NSERC). TRIUMF receives funding through a contribution agreement with the National Research Council of Canada (NRC). M.P. is supported in part by U.S. Department of Energy (Grant No. DE-SC0011842).

*mckeen@triumf.ca

†pospelov@umn.edu

¥nraj@triumf.ca

[1] E. Aprile et al. (XENON Collaboration), Phys. Rev. D 102, 072004 (2020).

[2] A. E. Robinson, arXiv:2006.13278.

[3] K. Kannike, M. Raidal, H. Veermäe, A. Strumia, and D. Teresi, arXiv:2006.10735 [Phys. Rev. D (to be published)].

[4] F. Takahashi, M. Yamada, and W. Yin, Phys. Rev. Lett. 125, 161801 (2020); L. Di Luzio, M. Fedele, M. Giannotti, F. Mescia, and E. Nardi, Phys. Rev. Lett. 125, 131804 (2020); G. Alonso-Álvarez, F. Ertas, J. Jaeckel, F. Kahlhoefer, and L. Thormaehlen, arXiv:2006.11243; C. Boehm, D. G. Cerdeno, M. Fairbairn, P. A. Machado, and A. C. Vincent, arXiv:2006.11250 [Phys. Rev. D (to be published)]; A. Bally, S. Jana, and A. Trautner, Phys. Rev. Lett. 125, 161802 (2020); D. Aristizabal Sierra, V. De Romeri, L. Flores, and D. Papoulias, Phys. Lett. B 809, 135681 (2020);
A. N. Khan, Phys. Lett. B 809, 135782 (2020); B. Fornal, P. Sandick, J. Shu, M. Su, and Y. Zhao, Phys. Rev. Lett. 125, 161804 (2020); M. Du, J. Liang, Z. Liu, V. Q. Tran, and Y. Xue, arXiv:2006.11949; Y. Chen, J. Shu, X. Xue, G. Yuan, and Q. Yuan, arXiv:2006.12447; Q.-H. Cao, R. Ding, and Q.-F. Xiang, arXiv:2006.12767; U. K. Dey, T. N. Maity, and T. S. Ray, arXiv:2006.12529; K. Harigaya, Y. Nakai, and M. Suzuki, Phys. Lett. B 809, 135729 (2020); L. Su, W. Wang, L. Wu, J. M. Yang, and B. Zhu, arXiv:2006.11837; N. F. Bell, J. B. Dent, B. Dutta, S. Ghosh, J. Kumar, and J. L. Newstead, Phys. Rev. Lett. 125, 161803 (2020); J. Buch, M. A. Buen-Abad, J. Fan, and J. S. C. Leung, arXiv:2006.12488; H. M. Lee, arXiv:2006.13183; G. Choi, M. Suzuki, and T. T. Yanagida, arXiv:2006.12348; G. Paz, A. A. Petrov, M. Tammaro, and J. Zupan, arXiv:2006.12462; K. Nakayama and Y. Tang, arXiv: 2006.13159; H. An, M. Pospelov, J. Pradler, and A. Ritz, arXiv:2006.13929; M. Baryakhtar, A. Berlin, H. Liu, and N. Weiner, arXiv:2006.13918; J. Bramante and N. Song, Phys. Rev. Lett. 125, 161805 (2020); C. Gao, J. Liu, L.-T. Wang, X.-P. Wang, W. Xue, and Y.-M. Zhong, Phys. Rev. Lett. 125, 131806 (2020); M. Lindner, Y. Mambrini, T. B. de Melo, and F.S. Queiroz, arXiv:2006.14590; L. Zu, G.-W. Yuan, L. Feng, and Y.-Z. Fan, arXiv:2006.14577; I. M. Bloch, A. Caputo, R. Essig, D. Redigolo, M. Sholapurkar, and T. Volansky, arXiv:2006.14521; H. An and D. Yang, arXiv:2006.15672.

[5] Y. Cui, M. Pospelov, and J. Pradler, Phys. Rev. D 97, 103004 (2018).

[6] D. McKeen, Phys. Rev. D 100, 015028 (2019); Z. Chacko, P. Du, and M. Geller, Phys. Rev. D 100, 015050 (2019).

[7] D. McKeen and A. E. Nelson, Phys. Rev. D 94, 076002 (2016).

[8] Z. Berezhiani, Lett. High Energy Phys. 2, 118 (2019).

[9] D. McKeen and M. Pospelov, arXiv:2003.02270.

[10] Z. Berezhiani and L. Bento, Phys. Rev. Lett. 96, 081801 (2006).

[11] Z. Berezhiani, Eur. Phys. J. C 76, 705 (2016).

[12] B. Fornal and B. Grinstein, Phys. Rev. Lett. 120, 191801 (2018).

[13] R. Allahverdi, P. S. B. Dev, and B. Dutta, Phys. Lett. B 779, 262 (2018); G. K. Karananas and A. Kassiteridis, J. Cosmol. Astropart. Phys. 09 (2018) 036.

[14] A. Czarnecki, W. J. Marciano, and A. Sirlin, Phys. Rev. Lett. 120, 202002 (2018).

[15] Z. Berezhiani, Eur. Phys. J. C 79, 484 (2019).

[16] Z. Tang, M. Blatnik, L. J. Broussard, J. H. Choi, S. M. Clayton et al., Phys. Rev. Lett. 121, 022505 (2018).

[17] M. Agostini et al. (Borexino Collaboration), Phys. Rev. Lett. 115, 231802 (2015).

[18] M. Pfützner and K. Riisager, Phys. Rev. C 97, 042501(R) (2018).

[19] D. McKeen, A. E. Nelson, S. Reddy, and D. Zhou, Phys. Rev. Lett. 121, 061802 (2018); G. Baym, D. H. Beck, P. Geltenbort, and J. Shelton, Phys. Rev. Lett. 121, 061801 (2018); T. F. Motta, P. A. M. Guichon, and A. W. Thomas, J. Phys. G 45, 05LT01 (2018); J. M. Cline and J. M. Cornell, J. High Energy Phys. 07 (2018) 081.

[20] G. Alimonti et al. (Borexino Collaboration), Phys. Lett. B 422, 349 (1998). 
[21] N. Arndt, Hydrosphere, in Encyclopedia of Astrobiology, edited by R. Amils, M. Gargaud, J. Cernicharo Quintanilla, H. J. Cleaves, W. M. Irvine, D. Pinti, and M. Viso (Springer, Berlin, Heidelberg, 2014), p. 1.

[22] R. K. Nesbet, J. Chem. Phys. 32, 1114 (1960); J. J. Sinai, J. Chem. Phys. 39, 1575 (1963); S. Kohda and S. Katagiri, Bull. Chem. Soc. Jpn. 46, 1428 (1973).

[23] C. W. Kern and M. Karplus, The water molecule, in The Physics and Physical Chemistry of Water, edited by F. Franks (Springer, New York, Boston, MA, 1972), pp. 21-91.

[24] E. Aprile et al. (XENON Collaboration), Phys. Rev. D 99, 112009 (2019).

[25] E. Aprile et al. (XENON Collaboration), Eur. Phys. J. C 80, 785 (2020).
[26] D. McKeen and N. Raj, Phys. Rev. D 99, 103003 (2019).

[27] B. M. Roberts and V. V. Flambaum, Phys. Rev. D 100, 063017 (2019).

[28] B. M. Roberts, V. A. Dzuba, V. V. Flambaum, M. Pospelov, and Y. V. Stadnik, Phys. Rev. D 93, 115037 (2016).

[29] E. Aprile, J. Aalbers, F. Agostini, M. Alfonsi, F. D. Amaro, M. Anthony, L. Arazi, F. Arneodo, C. Balan, P. Barrow et al., J. Cosmol. Astropart. Phys. 04 (2016) 027.

[30] X. Cui, A. Abdukerim, W. Chen, X. Chen, Y. Chen, B. Dong, D. Fang, C. Fu, K. Giboni, F. Giuliani et al., Phys. Rev. Lett. 119, 181302 (2017).

[31] B. Mount et al., arXiv:1703.09144.

[32] P. Agnes, I. Albuquerque, T. Alexander, A. Alton, G. Araujo, M. Ave, H. Back, B. Baldin, G. Batignani, K. Biery et al., Phys. Rev. D 98, 102006 (2018). 